\title{
Stress in Adolescents with a Chronically Ill Parent: Inspiration from Rolland's Family Systems-IIlness Model
}

\author{
D. S. Sieh • A. L. C. Dikkers • J. M. A. Visser-Meily • \\ A. M. Meijer
}

Published online: 6 July 2012

(C) The Author(s) 2012. This article is published with open access at Springerlink.com

\begin{abstract}
This article was inspired by Rolland's Family Systems-Illness (FSI) model, aiming to predict adolescent stress as a function of parental illness type. Ninety-nine parents with a chronic medical condition, 82 partners, and 158 adolescent children $(51 \%$ girls; mean age $=15.1$ years) participated in this Dutch study. The Dutch Stress Questionnaire for Children was used to measure child report of stress. Ill parents completed the Beck Depression Inventory. Children filled in a scale of the Inventory of Parent and Peer Attachment measuring the quality of parent attachment. Both parents filled in the ParentChild-Interaction Questionnaire-Revised. We conducted multilevel regression analyses including illness type, the ill parent's depressive symptoms, family functioning (quality of marital relationship, parent-child interaction, and parent attachment), and adolescents' gender and age. Four regression analyses were performed separately for each illness type as defined by disability (Model 1), and onset (Model 2), course (Model 3), and outcome of illness (Model 4). In all models, higher adolescent stress scores were linked to lower quality of parent-child interaction and parent attachment, and adolescents' female gender. The four models explained approximately $37 \%$ of the variance in adolescent stress
\end{abstract}

D. S. Sieh • A. L. C. Dikkers • A. M. Meijer

Research Institute of Child Development and Education, University of Amsterdam, the Netherlands, Nieuwe Prinsengracht 130, 1018 VZ Amsterdam, the Netherlands

A. L. C. Dikkers

e-mail: Anna.Dikkers@student.uva.nl

A. M. Meijer

e-mail: A.M.Meijer@uva.nl

J. M. A. Visser-Meily

Centre of Excellence in Rehabilitation Medicine, Rehabilitation Centre De Hoogstraat, Rembrandtkade 10, 3583 TM Utrecht, the Netherlands

e-mail: j.m.a.visser-meily@xs4all.nl

D. S. Sieh $(\bowtie)$

Department of Education, University of Amsterdam, Nieuwe Prinsengracht 130, 1018 VZ Amsterdam, the Netherlands

e-mail: D.S.Sieh@uva.nl 
between individuals and $43-44 \%$ of the variance in adolescent stress between families. Adolescent stress was not related to parental illness type. Our results partially supported the FSI model stating that family functioning is essential in point of child adjustment to parental illness. In the chronic stage of parental illness, adolescent stress does not seem to vary depending on illness type.

Keywords Chronic medical condition $\cdot$ Adolescent $\cdot$ Stress $\cdot$ Family-systems illness model

\section{Introduction}

Children of parents with a chronic medical condition (CMC) are at an increased risk for developing health-related and social-emotional problems, such as somatic complaints, social isolation, and excessive concern to acquire an illness themselves (Compas, 1994; Earley and Cushway 2002; Faulkner and Davey 2002; Pedersen and Revenson 2005). Also more recent evidence suggests that these children show internalizing problems (e.g., anxiety and depressed mood) and externalizing problems, that is, aggressive and rule-breaking behavior (Ivarsson et al. 2002; Sieh et al. 2010a). Children with parental CMC also display elevated stress levels (Pedersen and Revenson 2005; Sieh et al. 2010b). Stress in children presumably acts as a mediator between illness-related factors and child outcomes, and it is therefore an important variable to examine (Pakenham and Bursnall 2006; Pedersen and Revenson 2005). We choose adolescents as target group because they seem to be especially vulnerable to developmental problems and may adopt more caregiving responsibilities than latency-aged children (Korneluk and Lee 1998; Sieh et al. 2010a). Hence, this study will focus on adolescent stress.

According to the Family Systems-Illness (FSI) model of Rolland (1987, 1999), adolescent stress depends on specific illness-related factors, resulting in a differentiation into illness type depending on the diagnosis (Compas et al. 1994; Sieh et al. 2010a). The FSI model classifies CMC's as a function of illness type and family functioning. Illness type can be categorized based on the presence of disability (nondisabling versus disabling), its onset (gradual versus acute), its course (progressive, episodic, or constant), its outcome (fatal versus non-fatal), and its time stage (beginning versus terminal). Disability implicates problems with activities of daily living, and communicative and cognitive abilities. Disability of parents appears to be associated with elevated stress levels in the family (Pedersen and Revenson 2005; Rolland 1987, 1999; Verhaeghe et al. 2005). Concerning onset, diseases can have an acute onset, forcing the family to adapt in a short period of time, which often causes high stress levels directly after the diagnosis. Conversely, diseases can be qualified by a gradual onset, requiring continuous adjustment. The course of a chronic illness varies depending on the diagnosis and may be categorized based on the pattern of expected trajectory. A progressive illness increases in severity, inducing cumulative responsibilities for family caretakers over time, which is related to elevated stress levels in family members. An episodic illness is represented by exacerbations and remissions that require flexibility of all family members. Constant illnesses are often marked by an acute onset, implicating that the amount of illness-related stressors 
remains the same after recovery (Pedersen and Revenson 2005; Rolland 1987, 1999). Finally, the illness outcome is characterized by the possibility of death or shortened life span versus non-fatal conditions. The most important element is the initial doubt whether a disease will cause death (Rolland and Williams 2005). It is crucial to add that the FSI model considers illness type in relation to the illness stage (crisis, chronic stage, and terminal stage) and, in turn, in relation to family functioning. Accordingly, the stressors associated with the crisis stage of a less severe condition could easily generate more strain on a family system than the chronic phase of a more serious condition.

It is well-documented that $\mathrm{CMC}$ has a tremendous impact on psychological characteristics of ill parents, especially in terms of an increased risk for depression (Visser-Meily and Meijer 2006). For example, in the chronic stage of stroke, depression is a hidden issue that is linked to disability in parents (Van de Port et al. 2007). Similarly, a longitudinal study on children of stroke patients ascertained that depressive symptoms of ill parents predict long-term stress in children (Sieh et al. 2010b). Parental depression is often accompanied by decreased emotional availability of the parent and may directly and indirectly affect the quality of family relationships (Faulkner and Davey 2002). This study also takes the level of ill parents' depressive symptoms into account because of the strong association with family functioning.

The FSI model proposes that adolescent stress is associated with family functioning (Rolland 1987, 1999; Rolland and Williams 2005). In this study, family functioning is conceptualized by means of three interpersonal variables: quality of marital relationship, quality of parent-child interaction, and quality of parent attachment. Previous research revealed that the quality of marital relationship decreases following parental stroke. Two months after discharge from the rehabilitation center, it correlated with long-term stress in children (Sieh et al. 2010b). Researchers are inconclusive whether the relationship between quality of marital relationship and adolescent functioning is direct or bidirectional (Compas et al. 1994; Compas et al. 1996; Faulkner and Davey 2002; Sieh et al. 2010b; Veach 1999). However, they reached unanimity that the parent-child relationship should be evaluated by both parent and adolescent as the interpretations of this relationship might differ depending on the informant (Korneluk and Lee 1998; Hocking and Lochman 2005). Chronic parental illness affects the relationship between parents with $\mathrm{CMC}$ and their children. For example, children may adopt roles that are inappropriate for their age and feel overwhelmed by the sheer presence of household chores and caregiving tasks. The assumption of an adult role by a child, referred to as parentification, is an example of a possibly modified familial interaction pattern (Faulkner and Davey 2002; Meijer et al. 2007; Pedersen and Revenson 2005). All the same, family relationships can remain a source of strength and should be acknowledged as a protective buffer for adolescent stress (Carr and Springer 2010).

Besides, adolescents' gender and age may be associated with adolescent stress (Pedersen and Revenson 2005), but previous research delivered inconsistent results. Some studies found that girls have higher stress levels than boys (Sieh et al. 2010b; Welch et al. 1996). On the contrary, other studies reported no gender differences in child report of stress (Barkmann et al. 2007). Similarly, some studies suggested that older adolescents experience more stress than younger adolescents (Compas et al. 1994; Veach 1999), while other studies concluded that older adolescents experience 
less stress than younger adolescents (Faulkner and Davey 2002; Welch et al. 1996). It can be concluded that adolescents' gender and age should be controlled for with regard to child report of stress.

Inspired by the FSI model, the relation of adolescent stress to specific parental CMC's can be evaluated. Our sample concerns parents with a medical condition in the chronic stage, so we will examine in which regard the FSI model may be a useful explanatory framework apropos of child adjustment (adolescent stress) to chronic parental illness. To date, this has not been accomplished. According to the FSI model, chronic illnesses exhibit variability and without diagnostic differentiation, results may not apply to subsamples of children with a specific parental CMC. Therefore, the aim of this study is to investigate whether adolescents' stress level varies while controlling for parental illness type. The first question is whether adolescent stress is directly influenced by illness type (disability, onset, course, and outcome). Our second question is whether the size and significance of the associations with parental depression, family functioning, and child demographics depend on illness type.

We expected that children of parents with a disabling CMC showed more stress than children of parents with a non-disabling CMC. Similarly, parental CMC's with an acute onset were assumed to cause more stress in children than CMC's with a gradual onset. In addition, children of parents with a progressive CMC were supposed to have a higher stress level than children of parents with a constant CMC. Children of parents with a possibly fatal CMC were expected to have a higher stress level than children of parents with a non-fatal CMC. Children of families with high family functioning were assumed to show less stress than children of families with low family functioning (Rolland 1987, 1999).

\section{Method}

\section{Participants}

We included children between 10 and 20 years of age who lived together with at least one parent with a CMC. Healthy partners, if applicable, also participated. Parental CMC was defined as a disease or a traumatic injury impairing health, involving one or more organ systems and lasting 6 months or longer (Brown et al. 2007; Livneh and Antonak 2005). Children with severe somatic or psychiatric disorders, which were assessed by way of parent interviews and child report, were not eligible for participation. Of 116 families showing interest in participation, only 17 families were not part of the final sample, resulting in a high participation rate $(85.3 \%)$. Eight families dropped out without indicating a reason. One family indicated to perceive participation as a burden. The remaining 7 families could not participate because their children were too old, too young, or disabled, or because no parent had a CMC. In one case, no data of the parent with CMC were available and the data for this family were deleted from the analyses, leading to a sample of 99 families including 158 adolescents and 82 healthy parents (Table 1). Most families consisted of married parents or couples living together. Fifteen families were counted as single parent household, four of which were characterized by a long 
Table 1 Demographic characteristics of adolescents, ill, and healthy parents in families with parental $\mathrm{CMC}$

\begin{tabular}{lll}
\hline & $n(\%)$ & M (SD) \\
\hline Adolescents & 158 & \\
Gender (female) & $81(51.3)$ & \\
Age & & \\
Highest educational level & & \\
Primary education & $27(17.1)$ \\
Lower vocational education & $62(39.2)$ & \\
Intermediate vocational education & $23(14.6)$ \\
High school & $38(24.1)$ & \\
(Pre-)university education & $7(4.4)$ & \\
Ill parents & 99 & \\
Gender (female) & $67(67.7)$ & \\
Age & & \\
Highest educational level & & \\
Primary/lower education & $10(10.1)$ & \\
Intermediate vocational education & $29(29.3)$ \\
High school & $11(11.1)$ & \\
Pre-university or higher vocational education & $30(30.3)$ & \\
University & $14(14.1)$ & \\
Currently working & $37(37.4)$ & \\
Healthy parents & 82 & \\
Gender (female) & $32(31.7)$ \\
Age & & \\
Highest educational level & $11(12.2)$ & \\
Primary/lower education & $26(31.7)$ & \\
Intermediate vocational education & $6(7.3)$ & \\
High school & $25(30.5)$ & \\
Pre-university or higher vocational education & $14(14.1)$ & \\
University & $70(85.4)$ & \\
Currently working & & \\
\hline & & \\
\end{tabular}

distance relationship between the parents. In two families, both parents had a CMC and the data for the less disabled parent were excluded from analyses. All participants were Dutch. Most children followed lower vocational education. Over $40 \%$ of the parents with $\mathrm{CMC}$ had finished higher vocational education or university. Parental CMC included multiple sclerosis $(29.3 \%)$, rheumatoid arthritis $(19.2 \%)$, brain damage $(16.2 \%)$, muscle disease $(14.1 \%)$, spinal cord injury $(7.1 \%)$, inflammatory bowel disease $(6.1 \%)$, Parkinson disease $(5.1 \%)$, and diabetes type I with physical complications $(3.0 \%)$. The mean time since diagnosis was 12.6 years and ranged between 7 months and 49 years. 
Table 2 Number of parents with CMC as a function of illness type

\begin{tabular}{|c|c|c|c|c|c|}
\hline & & \multicolumn{2}{|l|}{ Non-disabling } & \multicolumn{2}{|l|}{ Disabling } \\
\hline & & Gradual onset & Acute onset & Gradual onset & Acute onset \\
\hline \multirow[t]{2}{*}{ Non-fatal } & Constant & 1 & 1 & 0 & $23^{\mathrm{a}}$ \\
\hline & Progressive & $15^{\mathrm{b}}$ & 0 & $18^{\mathrm{c}}$ & 1 \\
\hline \multirow[t]{2}{*}{ Potentially fatal } & Constant & 1 & 0 & 0 & 0 \\
\hline & Progressive & 0 & 0 & 0 & $39^{\mathrm{d}}$ \\
\hline
\end{tabular}

Examples of CMC's: ${ }^{a}$ multiple sclerosis, cystic fibrosis; ${ }^{b}$ rheumatoid arthritis, hereditary motor sensory neuropathy; ${ }^{\mathrm{c}}$ stroke, spinal cord injury, traumatic brain injury; ${ }^{\mathrm{d}}$ colitis ulcerosa. Disabling disease: expected problems with activities of daily living, and with communicative or cognitive activities like walking, dressing, and talking. Acute onset: onset of disease less than $1 \mathrm{~h}$, diagnosis easily made. Progressive course: disease increasing in severity. Possibly fatal: possibility of death or shortened life span

\section{Measures}

Illness Type A medical doctor in our team documented illness type in a spread sheet in accordance with the FSI model (Rolland 1987; Stehouwer et al. 2010; World Health Organization 2001), see Table 2. When a parent had more than one CMC, the categorization of illness into illness type was based on the worst possible outcome and disability. Because of the small sample size, we decided to dichotomize the scales of the classification system with the following scores: non-disabling (0) versus disabling (1), gradual onset (0) versus acute onset (1), constant course (0) versus progressive course (1), non-fatal (0) versus potentially fatal (1).

Adolescent Stress Adolescents filled in the Dutch Stress Questionnaire for Children (Hartong et al. 2003), a reliable self-report measure to determine global psychological stress (17 items; 4-point Likert scale from $1=$ not true for me at all to $4=$ completely true for me). In this study, Cronbach's alpha was $\alpha=0.87$.

Depressive Symptoms in Ill Parent's Depressive symptoms of parents with CMC, also referred to as parental depression, were determined with the Beck Depression Inventory (21 items; 4-point scale from $0=I$ do not feel like a failure to $3=I$ feel I am a complete failure as a person, $\alpha=0.87$ ) (Beck et al. 1961; Visser et al. 2006; Yin and Fan 2000).

Family Functioning Three scales were used to assess family functioning. The Interactional Problem Solving Inventory (IPSI) measuring perceived quality of marital relationship from Lange (1983) was filled in by both parents. Only 10 single parents with CMC did not fill in the IPSI. Where applicable, we calculated dyadic scores reflecting the quality of marital relationship for each family (17 items; 5-point Likert scale from 1 = exactly applicable to me/my partner to $5=$ absolutely not applicable to me/my partner; $\alpha=0.85$ ). To assess the quality of parent-child interaction, both parents completed the Parent-Child Interaction Questionnaire Revised from Lange 
(2001). Average scores were calculated to generate one parent-child interaction score for each family (21 items; 5-point Likert scale; 1 = completely inapplicable, $4=$ exactly applicable; $\alpha=0.87$ ). In addition, adolescents filled in three scales (communication, confidence, and alienation) of the Inventory of Parent and Peer Attachment (Nada Raja et al. 1992; Reitz 2004) about the father and the mother, and average scores were calculated to measure overall parent attachment (12 items; 4-point Likert scale from 1 = almost never or never to $4=$ almost always or always; $\alpha=0.88$ ).

Adolescents' Gender and Age Gender was scored as male (0) and female (1). We used the exact age with two decimals.

\section{Procedure}

Families with parental $\mathrm{CMC}$ were recruited through general health practitioners, health organizations, rehabilitation and community centers, hospitals, schools, and public places (e.g., libraries) across the Netherlands. Collaborating staff was instructed by the project manager and posted brochures and posters in waiting rooms and public spaces. Some of them also provided additional information and invited potential participants to take part in this study. Families had to contact the researchers by e-mail or phone to show their interest in participation. Research assistants visited the families at home to implement several questionnaires. Both parents and adolescents provided active informed consent. The ethical commission of the research institute of Child Development and Education of the University of Amsterdam approved this study.

\section{Data Analyses}

The research questions were answered by means of multilevel regression analyses which accounted for the fact that children within families have more similarities than children between families (Snijders and Bosker 1999). We used linear mixed modeling of SPSS, version 20.0. Adolescents acted as Level 1, while the family acted as Level 2. The nesting structure of adolescents and chronically ill parents is illustrated in Table 3.

Using $G^{*}$ Power (Buchner et al. 2009), we found that the power of this study was 0.89 , while correcting for nested data with the expectation of equal sample sizes in each group. A power value that large means that it was probable that we would find a

Table 3 Nesting structure of adolescents per marital status or living condition

\begin{tabular}{llllll}
\hline & \multicolumn{4}{l}{$\begin{array}{l}\text { Number of adolescents within } \\
\text { families }\end{array}$} \\
\cline { 2 - 6 } & 1 & 2 & 3 & 4 & Total \\
\hline Single parent home & 10 & 4 & 1 & 0 & 15 \\
Parents married/ living together & 41 & 34 & 8 & 1 & 84 \\
Total & 51 & 38 & 9 & 1 & 99 \\
\hline
\end{tabular}


statistically significant result when such a result actually exists. Due to a small sample size within many cells, no analysis could be performed including all illness types at once. Four analyses of illness type were conducted separately and included all other predictors in each analysis. In the first analysis (Model 1), the influence of disability was examined. Model 2 concerned the influence of illness onset (gradual versus acute). Model 3 included constant illnesses versus progressive illnesses. Model 4 examined non-fatal CMC's versus potentially fatal CMC's. We report the Akaike information criterion as a measure of the relative goodness of model fit. To illustrate comparable estimates, all independent variables were standardized.

Only $0.9 \%$ of the data from children and $3.5 \%$ of the data from parents were missing throughout the dataset. Data were missing completely at random (Little 1988), so we used expectation maximization to substitute missing values.

\section{Results}

Children were distributed among the following categories of parental illness type: 132 cases of disability versus 26 cases of no disability, 117 cases of gradual onset versus 41 cases of acute onset, 44 cases of constant course versus 114 cases of progressive course, and 92 cases of non-fatal outcome versus 66 cases of possibly fatal outcome.

The level of adolescent stress was comparable to that of children 3 years after parental stroke (Sieh et al. 2010b). Half of the ill parents (50.5\%) displayed scores that indicated mild $(29.3 \%)$, moderate $(10.1 \%)$, or severe depression $(11.1 \%)$. The mean quality of marital relationship was under the cut-off score (68.5 points) and $69.5 \%$ of the parents scored below the cut-off score, indicating relatively poor marital functioning. The average scores for quality of parent-child interaction were very close to those of the normal population (mean $=88.82, S D=6.59$; Lange 2001), see Table 4.

All independent variables (parental depression; quality of marital relationship, parent-child interaction, and attachment; adolescent gender and age) had a significant correlation with adolescent stress, but only quality of parent attachment showed a large correlation and all other correlations with adolescent stress ranged between small and medium (Cohen, 1992), see Table 5. Most correlations between the predictors were significant, one of which was large in size, indicating that higher

Table 4 Descriptive statistics for adolescent stress, parental depression, quality of marital relationship, quality of parent-child interaction and quality of parent attachment

\begin{tabular}{lll}
\hline & Mean $(S D)$ & Range \\
\hline Adolescent stress & $34.66(8.07)$ & $17.0-63.0$ \\
Parental depression & $12.19(7.70)$ & $1.0-34.0$ \\
Quality of marital relationship & $62.01(9.96)$ & $34.0-78.0$ \\
Quality of parent-child interaction & $88.99(10.69)$ & $49.0-104.0$ \\
Quality of parent attachment & $38.89(5.59)$ & $15.5-48.0$ \\
\hline
\end{tabular}


Table 5 Correlations between Adolescent Stress, Parental Depression, Family Functioning, and Adolescents' Gender and Age

\begin{tabular}{lllllll}
\hline & 1 & 2 & 3 & 4 & 5 & 6 \\
\hline 1 Adolescent stress & - & & & & & \\
2 Parental depression & $0.24^{* *}$ & - & & & & \\
3 Quality of marital relationship & $-0.20^{*}$ & $-0.67^{* *}$ & - & & & \\
4 Quality of parent-child interaction & $-0.30^{* *}$ & $-0.32^{* *}$ & $0.42^{* *}$ & - & & \\
5 Quality of parent attachment & $-0.56^{* *}$ & $-0.31^{* *}$ & $0.36^{* *}$ & $0.29^{* *}$ & - & \\
6 Adolescents' gender & $0.19^{*}$ & -0.04 & 0.01 & 0.05 & -0.03 & - \\
7 Adolescents' age & $0.25^{* *}$ & -0.04 & -0.04 & -0.10 & $-0.25^{* *}$ & 0.01 \\
\hline
\end{tabular}

${ }^{*} p<0.05 .{ }^{* *} p<0.01$

quality of marital relationship was strongly associated with lower parental depression scores. The size of the other correlations between family functioning variables and parental depression, and within family functioning variables was medium. Adolescents' gender and age showed three small correlations, that is, girls and older children comparatively reported more stress and older children reported a lower quality of parent attachment.

An empty model was estimated, with only the random intercept and the family as the grouping variable (Table 6). The intra-class correlation coefficient $(\mathrm{ICC}=0.29)$ and the deviance ratio $\left[\chi^{2}(1)=4.47, p<0.05\right]$ indicated that a model with a random intercept fitted the data better than a model that did not allow for random variability. The ICC can be interpreted as such that two random children in the same random family shared $29 \%$ of the variability.

First, Model 1 was tested while controlling for the presence of disability. Quality of parent-child interaction and parent attachment, and adolescents' gender were significantly linked to adolescents' stress level, see Table 6. Disability of ill parents was not related to adolescent stress. Model 2 concerned the illness onset characterized as either gradual or acute. Estimates for quality of parent-child interaction and parent attachment, and adolescents' gender were statistically significant. Illness onset was not associated with adolescent stress. Model 3 controlled for constant and progressive CMC's. Quality of parent-child interaction and parent attachment, and adolescents' gender displayed significant associations with adolescent stress. Illness course was unrelated to adolescent stress. Model 4 included the outcome of the illness (non-fatal versus possibly fatal). Again, quality of parent-child interaction and parent attachment, and adolescents' gender had significant relationships with adolescent stress. Illness outcome was not linked to adolescent stress.

In sum, the results demonstrated that independent of illness type, higher quality of parent-child relationships and adolescents' female gender were connected to elevated adolescent stress. The Akaike Information Criterion of Model 1 to Model 4 showed that the model fit improved compared to the empty model. The four models explained approximately $37 \%$ of the variance in adolescent stress between individuals and 43$44 \%$ of the variance in adolescent stress between families. 


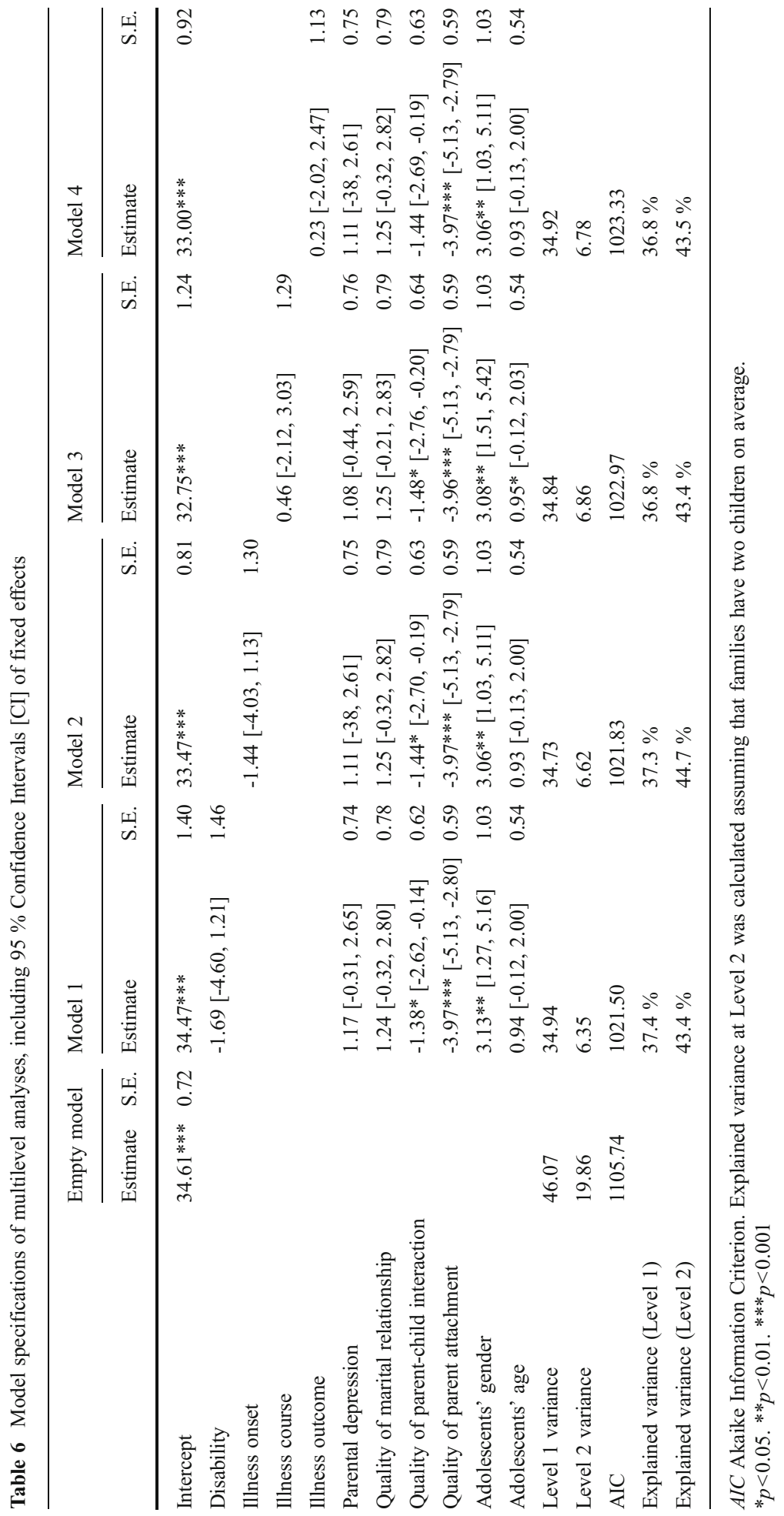




\section{Discussion}

This study evaluated the predictive power of illness type, the ill parent's depressive symptoms, family functioning, and adolescents' gender and age on child report of stress in a sample of parents with a medical condition in the chronic stage. First, illness type in the chronic stage of parental illness did not appear to affect adolescent stress scores. Second, the size and significance of the estimates for parental depression, family functioning and child demographics did not depend on illness type. Hence, the results cannot confirm the expectation based on the FSI model suggesting a significant role of illness type with respect to child report of stress. Contrary to our hypotheses, not illness type but mainly family functioning and adolescents' gender were directly related to adolescent stress. All variances explained by the models were moderate, meaning that we identified important risk and protective factors for adolescent stress at the individual level (i.e., quality of parent attachment and adolescent gender) and at family level (i.e., quality of parent-child interaction). In support of the FSI model, strong evidence was found that the parent-child relationship is a crucial determinant of adolescent stress.

Unexpectedly, the relationship between parental depression and adolescent stress was not significant in regression analyses. On the contrary, the empirical and theoretical basis suggests that depressive symptoms of ill parents often emerge over time (Sieh et al. 2010b), eminently in more debilitating and progressive conditions. In accordance, Rolland (1987; 1999) stated that disabling and possibly fatal diseases have the strongest impact on family functioning. We did not find support for this assumption and can only present a small to medium positive correlation between parental depression and adolescent stress. Possibly, other mediating variables (e.g., parenting and coping variables) are involved in the relationship between ill parents' depressive symptoms and adolescent stress (D'Onofrio and Lahey 2010; Sieh et al. 2010b).

Similarly, high quality of marital relationship was merely correlated with elevated adolescent stress. However, no significant relationship was found between marital functioning and adolescent stress when we controlled for illness type, parent-child interaction, parent attachment, and demographics. Research is inconsistent about the influence of quality of marital relationship on adolescents' functioning (Compas et al. 1994, 1996; Faulkner and Davey 2002; Sieh et al. 2010b; Veach 1999). In our sample, marital functioning was poor, resulting in lower variance, which may obstruct significant results. A longitudinal study on families of stroke patients showed that marital functioning progressively decreased under the level of adequate functioning. Marital functioning during 3 years post-stoke was just incidentally associated with long-term stress in adolescents (Sieh et al. 2010b). A possible explication for inconsistent findings in the field is that marital functioning and child adjustment are bidirectional and reinforce each other through a mediating variable such as parenting and parent attachment (D’Onofrio and Lahey 2010).

High quality of parent-child interaction and especially high quality of parent attachment were associated with lower levels of adolescent stress, pointing to a potential protective mechanism in child adjustment to parental CMC. Notably, the estimates for parent attachment were the highest. As yet, previous research has attested the paramount importance of family functioning in child adjustment to parental CMC (Carr and Springer 2010; Hocking and Lochman 2005; Korneluk and Lee 1998; Sieh et al. 
2010a). Our study extends previous knowledge, affirming that especially variables evaluating the parent-child relationship show high predictive power for adolescent stress. This suggests that rehabilitation staff can be recommended to give attention to the parenting role, how the parenting role may change as a result of $\mathrm{CMC}$, and how parental CMC affects the parent-child relationship. For example, parents may need reassurance when they do a good job. They could also benefit from advice about how to talk to children about their condition and its impact on family life. In addition, the results substantiate that the parent-child relationship should be evaluated by both parents and adolescents who may evaluate the mutual relationship differently (Brumariu and Kerns 2010; Korneluk and Lee 1998; Meijer et al. 2007).

Moreover, girls appear to be more susceptible to stress than boys, which is in line with most research in the field (Sieh et al. 2010a; b; Verhaeghe et al. 2005). The estimate for gender was highly significant, bringing into view that girls have different sensitivities than boys. It may be hypothesized that girls could benefit from stress management more than boys. Lastly, adolescents' age was unrelated to child report of stress in the regression analysis and we found a small positive correlation. Previous research is inconsistent about the influence of adolescents' age on adolescents' wellbeing (Faulkner and Davey 2002; Pedersen and Revenson 2005; Sieh et al. 2010a). More research is needed for distinct conclusions regarding the effects of age on adolescent stress in this specific group.

This study had some limitations. Our sample size was small for the amount of cases per illness type except in Model 1 (including disability). A related restraint is that we had to dichotomize the scales of illness type and could not account for progressive illness course or fatal outcomes, potentially altering the results. For example, illness outcome may have been unrelated to adolescent stress due to the omission of illness fatality. Similarly, because of the sample size, we had to ignore the interactive effects of one versus two parents with a disability, and one CMC versus more than one CMC. Moreover, it should be borne in mind that we examined CMC in a sample of Western culture. The results could have looked differently using data from a sample of non-Western culture because of distinctive cultural views and practices affecting health and health behaviors (Carr and Springer 2010).

An important strength of this study lies in the inspiration from the FSI model in which medical information is combined with information about family functioning of the parent and adolescent. In addition, this study recognized factors that emerged as influential predictors in previous research, including reports of the parent-child relationship from parents and adolescents. As recommended (D'Onofrio and Lahey 2010), we took into consideration family characteristics at multiple levels (demographic, psychological, and relational variables from several informants). Finally, the use of multilevel analyses is an advancement as grouping according to families led to a considerable similarity between adolescents within the same family.

Our study did not aim to generate specific recommendations for the clinical practice, but possible interventions should not go unmentioned. In an extensive review, Weihs et al. (2002) stratified studies by specific CMC's and formulated three general goals for interventions for families affected by CMC. Recommendations include helping families cope with illness-related stressors, mobilizing the family support system, and minimizing interpersonal hostility and adverse effects of illnessrelated trauma. Carr and Springer (2010) reviewed research on families and health 
between 2000 and 2009. In line with our findings stressing the importance of family relationships, Carr and Springer identify interpersonal relationships as potentially modifiable factors. Interventions should aim to encourage healthy relationships through parent education, conflict and stress management, communicative training, and health promotion. For example, cognitive-behavioral stress management training has shown to benefit mental health, social interaction, and family functioning in adolescents with parental CMC (Keypour et al. 2011).

Future studies should incorporate all elements of the FSI model, taking into account the stage of an illness consisting of crisis, chronic stage, and terminal stage (Rolland 1987, 1999). Stages of individual family members and family life cycles also need to be considered. Our data were collected in the chronic stage, making it impossible to compare the stress level in different illness stages. With a larger and more diverse illness sample, the FSI model can be fully applied and the hypotheses can be formulated in more complex ways. Notably, the transitions between time stages are crucial in relation to the other illness types. For instance, the stressors associated with the crisis stage of a less severe condition could easily generate more strain on a family system than the chronic phase of a more serious condition. Similarly, the hypothesis that acute onset CMC is associated with more stress in adolescents than a gradual onset CMC could depend on the time stage in which families are investigated. In case of parental stroke (acute onset) relative to multiple sclerosis (gradual onset), the hypothesis may initially hold. Yet, if the measurement occurred several years later, families dealing with a stroke would show considerable improvement over time (Visser-Meily et al., 2009), potentially decreasing the experience of stress (Sieh et al. 2010b). Besides, the presence of disability depends on the stage of illness. To illustrate, multiple sclerosis can be non-disabling in the beginning and cause severe disability a decade later. These complex relationships between illness type and illness stage could lead to interesting results from forthcoming longitudinal studies (Rolland 1987, 1999; Rolland and Williams 2005). Further, studies could focus on the mediating and moderating role of stress and family characteristics in the development of problem behavior in adolescents. D'Onofrio and Lahey (2010) have conducted a decade review on biosocial studies focusing on family processes. They emphasize the need to be thoughtful of bidirectional influences among family members and the wider social and biological system, embodying interactions between environmental and biological factors (e.g., stress hormones like cortisol) over time.

Lastly, we investigated medical and psychological factors, whereas theories and models are usually developed from a specific perspective, for example, a medical perspective. To increase our understanding of the complex FSI model, it is necessary to better understand family relationships and the interplay between all components of the model. This can be accomplished by means of transdisciplinary theories and models, for example, the social model of disability (Tate and Pledger 2003). According to that model, the perceived stressfulness does not necessarily result from the parent's physical disability but rather from interpersonal experiences and environmental mismatches that functional limitations can precipitate.

In conclusion, this study increases our understanding of the FSI model and provides insight into protective and risk factors for adolescent stress in the chronic stage of parental medical condition. We found some support for the FSI model in the 
sense that family functioning is associated with adolescent stress, but this association does not seem to depend on parental illness type in the chronic stage. It is valuable to incorporate interpersonal family variables and adolescents' gender in predictive models of adolescent stress. Rehabilitation staff is recommended to consider how parental CMC affects the parent-child relationship and how family relationships can be boosted, offering interventions such as stress and conflict management.

Acknowledgments This study is part of a $\mathrm{PhD}$ program which is supported by a grant from the Netherlands Organization for Health Research and Development (ZonMw). The authors thank all participating families. We further thank Bonne J.H. Zijlstra, PhD, for his statistical assistance and Frederik G. Dikkers, $\mathrm{MD}, \mathrm{PhD}$, for correction of earlier versions of this manuscript.

Open Access This article is distributed under the terms of the Creative Commons Attribution License which permits any use, distribution, and reproduction in any medium, provided the original author(s) and the source are credited.

\section{References}

Barkmann, C., Romer, G., Watson, M., \& Schulte-Markwort, M. (2007). Parental physical illness as a risk for psychosocial maladjustment in children and adolescents: epidemiological findings from a national survey in Germany. Psychosomatics, 48, 476-481.

Beck, A. T., Ward, C. H., Mendelson, M., Mock, J., \& Erbaugh, J. (1961). An inventory for measuring depression. Archives of General Psychiatry, 4, 561-571.

Brown, R. T., Daly, B. P., \& Rickel, A. U. (2007). Chronic illness in children and adolescents. Cambridge: Hogrefe \& Huber Publishers.

Brumariu, L. E., \& Kerns, K. A. (2010). Parent-child attachment and internalizing symptoms in childhood and adolescence: a review of empirical findings and future directions. Development and Psychopathology, 22, 177-203.

Buchner, A., Erdfelder, E., Faul, F., \& Lang, A. G. (2009). G*Power version 3.1.2. Kiel: University of Kiel.

Carr, D., \& Springer, K. W. (2010). Advances in families and health research in the 21 st century. Journal of Marriage and Family, 72, 743-761.

Cohen, J. (1992). A power primer. Psychological Bulletin, 112, 155-159.

Compas, B. E., Worsham, N. L., Epping-Jordan, J. E., Grant, K. E., Mireault, G., Howell, D. C., \& Malcarne, V. L. (1994). When mom or dad has cancer: markers of psychological distress in cancer patients, spouses, and children. Health Psychology, 13, 507-515.

Compas, B. E., Worsham, N. L., Ey, S., \& Howell, D. C. (1996). When mom or dad has cancer II: coping, cognitive appraisals, and psychological distress in children of cancer patients. Health Psychology, 15, $167-175$.

D’Onofrio, B. M., \& Lahey, B. B. (2010). Biopsychosocial influences on the family: a decade review. Journal of Marriage and Family, 72, 762-782.

Earley, L., \& Cushway, D. (2002). The parentified child. Clinical Child Psychology and Psychiatry, 7, 163178.

Faulkner, R. A., \& Davey, M. (2002). Children and adolescents of cancer patients: the impact of cancer on the family. The American Journal of Family Therapy, 30, 63-72.

Hartong, I., Krol, M., Maaskant, A., Plate, A., \& Schuszler, D. (2003). Psst.. Are you asleep? Study on the quality of sleep. Amsterdam: University of Amsterdam.

Hocking, M. C., \& Lochman, J. E. (2005). Applying the transactional stress and coping model to sickle disease and insulin-dependent diabetes mellitus: identifying psychosocial variables related to adjustment and intervention. Clinical Child and Family Psychology Review, 8, 221-246.

Ivarsson, T., Gillberg, C., Arvidsson, T., \& Broberg, A. G. (2002). The Youth Self-Report (YSR) and the Depression Self-Rating Scale (DSRS) as measures of depression and suicidality among adolescents. European Child and Adolescent Psychiatry, 11, 31-37. 
Keypour, M., Arman, S., \& Maracy, M. R. (2011). The effectiveness of cognitive behavioral stress management training on mental health, social interaction and family function in adolescents of families with one Human Immunodeficiency Virus (HIV) positive member. Journal of Research in Medical Science, 16, 741-9.

Korneluk, Y. G., \& Lee, C. M. (1998). Children's adjustment to parental physical illness. Clinical Child and Family Psychology Review, 1, 179-193.

Lange, A. (1983). Interactionele Probleem Oplossings Vragenlijst (IPOV) [Interactional Problem Solving Inventory (IPSI)]. Deventer: Van Loghum Slaterus.

Lange, A. (2001). De Ouder-Kind Interactie Vragenlijst - Revised, OKIV-R [Parent-Child Interaction Questionnaire Revised]. Houten: Bohn Stafleu Van Loghum.

Little, R. J. A. (1988). A test of missing completely at random for multivariate data with missing values. Journal of the American Statistical Association, 83, 1198-1202.

Livneh, H., \& Antonak, R. F. (2005). Psychological adaptation to chronic illness and disability: a primer for counselors. Journal of Counseling and Development, 83, 12-20.

Meijer, A. M., Smit, M., Stams, G. J., Visser-Meily, J. M. A., \& Port, I. van de (2007). Chronische ziekte van een ouder en verandering in ouder-kind contact [Chronic illness of a parent and change in parentchild contact]. Intern document University of Amsterdam, the Netherlands.

Nada Raja, S., McGee, R., \& Stanton, W. R. (1992). Perceived attachments to parents and peers and psychological well-Being in adolescence. Journal of Youth and Adolescence, 12, 471-485.

Pakenham, K. I., \& Bursnall, S. (2006). Relations between social support, appraisal and coping and both positive and negative outcomes for children of a parent with multiple sclerosis and comparisons with children of healthy parents. Clinical Rehabilitation, 20, 709-723.

Pedersen, S., \& Revenson, T. A. (2005). Parental illness, family functioning, and adolescent well-being: a family ecology framework to guide research. Journal of Family Psychology, 19, 404-409.

Reitz, E. (2004). Problem behavior during early adolescence and child, parent, and friends effects. A longitudinal study. Amsterdam: University of Amsterdam.

Rolland, J. S. (1987). Chronic illness and the life cycle: a conceptual framework. Family Process, 26, 203221.

Rolland, J. S. (1999). Parental illness and disability: a family systems framework. Journal of Family Therapy, 21, 242-266.

Rolland, J. S., \& Williams, J. K. (2005). Toward a biopsychosocial model for 21 st-century genetics. Family Process, 44, 3-24.

Sieh, D. S., Meijer, A. M., Oort, F. J., Visser-Meily, J. M. A., \& van der Leij, D. A. V. (2010). Problem behavior in children of chronically ill parents: a meta-analysis. Clinical Child and Family Psychology Review, 13, 384-397.

Sieh, D. S., Meijer, A. M., \& Visser-Meily, J. M. A. (2010). Risk factors for stress in children after parental stroke. Rehabilitation Psychology, 55, 391-397.

Snijders, T. A. B., \& Bosker, R. J. (1999). Multilevel analysis: An introduction to basic and advanced multilevel modeling. London: Sage.

Stehouwer, C. D. A., Koopmans, R. P., \& van der Meer, J. (2010). Interne geneeskunde [Internal medicine]. Houten: Bohn Stafleu van Loghum.

Tate, D. G., \& Pledger, D. C. (2003). An integrative conceptual framework of disability: new directions for research. American Psychologist, 58, 289-295.

Van de Port, I. G. L., Kwakkel, G., Schepers, V. P. M., Heinemans, C. T. I., \& Lindeman, E. (2007). Is fatigue an independent factor associated with activities of daily living, instrumental activities of daily living and health-related quality of life in chronic stroke? Cerebrovascular Diseases, 23, 40-5.

Van de Port, I., Visser-Meily, A., Post, M., \& Lindeman, E. (2007). Long-term outcome in children of patients after stroke. Journal of Rehabilitation Medicine, 39, 703-707.

Veach, T. A. (1999). Families of adult cancer patients. Journal of Family Psychotherapy, 10, 43-59.

Verhaeghe, S., Defloor, T., \& Grypdonck, M. (2005). Stress and coping among families of patients with traumatic brain injury: a review of the literature. Journal of Clinical Nursing, 14, 1004 1012 .

Visser, M., Leentjes, A. F. G., Marinus, J., Stiggelbout, A. M., \& van Hilten, J. J. (2006). Reliability and validity of the Beck Depression Inventory in patients with Parkinson's disease. Movement Disorders, 21, 668-672.

Visser-Meily, J. M. A., \& Meijer, A. M. (2006). Stroke, consequences for the family and recommendations for rehabilitation. In D. M. Devore (Ed.), Parent child relations: New research (pp. 157-174). Hauppauge: Nova Science. 
Visser-Meily, J. M. A., Post, M. W. M., Van de Port, I., Maas, C. J. M., Forstberg-Wärleby, G., \& Lindeman, E. (2009). Psychosocial functioning of spouses of patients with stroke from initial inpatient rehabilitation to 3 years poststroke: course and relations with coping strategies. Stroke, 40, 1399-1404.

Weihs, K., Fisher, L., \& Baird, L. (2002). Families, health and behavior. Families, Systems, \& Health, 20, $7-46$.

Welch, A. S., Wadsworth, M. E., \& Compas, B. E. (1996). Adjustment of children and adolescents to parental cancer: parents' and children's perspectives. Cancer, 77, 1409-1418.

World Health Organization. (2001). International classification of functioning, disability and health. Geneva: World Health Organization.

Yin, P., \& Fan, X. (2000). Assessing the reliability of Beck Depression Inventory scores: reliability generalization across studies. Educational and Psychological Measurement, 60, 201-223. 